\title{
The Simon effect and handedness: Evidence for a dominant-hand attentional bias in spatial coding
}

\author{
SANDRO RUBICHI \\ Università di Modena e Reggio Emilia, Reggio Emilia, Italy \\ and \\ ROBERTO NICOLETTI \\ Università di Bologna, Bologna, Italy
}

\begin{abstract}
In two experiments, the relation between handedness and the size of the Simon effect in each visual hemifield was investigated. Experiment 1 showed that the Simon effect was larger in the right visual hemifield in right-handers and in the left visual hemifield in left-handers, whereas ambidextrous individuals showed a symmetric Simon effect. In Experiment 2, participants performed the same Simon task as in Experiment 1, but with their hands crossed. The right- and left-handed groups showed a reversed pattern of results with respect to Experiment 1. We explained this phenomenon as a part of a more general account in which perception and action are embedded in a perception-for-action system. In this system, an attentional bias originating from the field of operation of the dominant hand would be at the basis of the relationship between the asymmetry of the Simon effect and handedness.
\end{abstract}

In choice reaction time (RT) tasks, the spatial relation between stimulus and response affects overt behavior even when space is not relevant for the task. In the socalled Simon task (Simon \& Rudell, 1967; see Hommel \& Prinz, 1997, and Lu \& Proctor, 1995, for reviews), for example, participants typically make choice responses to a nonspatial stimulus attribute (e.g., form or color) with keys arranged on the left and right. Stimulus locations have the same (spatial) arrangement as response locations, lying horizontally, for example. Accuracy is better and RTs shorter when the irrelevant stimulus location corresponds to the response location (corresponding condition) than when it does not (noncorresponding condition). Accounts of the Simon effect often invoke dual-route models, in which it is assumed that the onset of the imperative stimulus could activate both a direct and an indirect route, which in turn activate a response in independent and parallel fashions (see, e.g., De Jong, Liang, \& Lauber, 1994; Kornblum, Hasbroucq, \& Osman, 1990). The fast direct route processes the task-irrelevant spatial stimulus dimension, so that the response that corresponds to stimulus location could be automatically primed irrespective of task instructions. Through the slower, indirect route, in contrast, the task-relevant nonspatial stimulus feature activates the response indicated by the instructions. The cor-

This study was supported by grants from MIUR. We thank Richard Abrams, Cristina Iani, Jason Ivanoff, and Robert Proctor for their very helpful comments on an earlier draft of this work. Correspondence concerning this article should be addressed to S. Rubichi, Dipartimento di Scienze Sociali, Cognitive e Quantitative, Università di Modena e Reggio Emilia, Viale Allegri, 9, 42100 Reggio Emilia, Italy (e-mail: rubichi@ unimore.it). responding trials lead to more efficient performance because both routes activate the same response. Performance on noncorresponding trials, in contrast, is slower and less accurate because competing responses are activated at the response selection stage, thus generating a conflict that must be resolved before response execution.

Psychophysiological evidence supporting the dual-route notion comes from event-related brain potential studies. Following the presentation of a lateral target stimulus, a component is evident over the motor cortex, indicating the activation of a response that corresponds to stimulus location. This component, called lateralized readiness potential, is recognized as an index of motor preparation. With regard to the Simon task, two activation components associated with the selection of responses have been consistently found (see, e.g., De Jong et al., 1994; Eimer, 1995; Valle-Inclán, 1996). An initial component is thought to reflect the location-based priming of the corresponding response, whereas a later component would reflect the rule-based activation of the response specified by the task instructions. The neural mechanisms underlying the early response-priming process have also been confirmed by means of electromyographic measures (Hasbroucq, Possamaï, Bonnet, \& Vidal, 1999) and by transcranial magnetic stimulation and motor-evoked potentials (Stürmer, Siggelkow, Dengler, \& Leuthold, 2000).

In the present study, we were interested in exploring the relationship between spatial coding and handedness in a Simon-like task. Useful insights on this topic can be derived by the seminal study of Simon and Rudell (1967) in which the Simon effect was accidentally discovered. The authors designed an auditory Simon task because they were originally interested in the phenomenon of hemi- 
spheric dominance for speech. Right- and left-handers were enrolled, and the authors expected to find an interaction between ear stimulated and handedness. Instead, they found an interaction between stimulus and response locations (i.e., the Simon effect). Interestingly, however, the interaction between handedness and response position was also significant, indicating that right-handers had a larger Simon effect in the right hemifield whereas left-handers had a larger Simon effect in the left hemifield. This interaction was not systematically explored in subsequent studies, probably because the Simon effect is considered a phenomenon arising from abstract spatial codes of stimuli and responses. ${ }^{1}$ Indeed, according to the coding hypothesis put forward by Wallace (1971), the Simon effect does not depend on the absolute locations of stimuli or responses. The author found that the Simon effect was of the same magnitude when the left/right keypress responses to the stimuli were executed either with uncrossed effectors (left hand on left key and right hand on right key) or with crossed effectors (left hand on right key and right hand on left key). Interestingly, in this and a subsequent study (Wallace, 1972), Wallace enrolled only right-handers. From the data reported, the magnitude of the Simon effect was larger for the right than for the left visual hemifield in the uncrossed-hand condition, and it was larger for the left than for the right visual hemifield in the crossed-hand condition. As we mentioned above, however, these studies were conducted to support the coding hypothesis of the Simon effect. Later, Umiltà and Nicoletti (1985) demonstrated that the spatial codes underlying the Simon effect can be based on relative locations. A normal Simon effect was obtained when both stimulus locations and both response locations were to the left or to the right of the body midline. In addition, there is evidence that multiple spatial codes can be formed for a given stimulus on the basis of static attributes of the display with different frames of reference (Lamberts, Tavernier, \& d'Ydewalle, 1992; Roswarski \& Proctor, 1996; Umiltà \& Liotti, 1987), such as hemispace (left or right of center), visual hemifield within hemispace (to the left or right of a fixation cross located in a hemispace), and relative position within a hemifield (left or right).

Interestingly, both the Simon effect and handedness have been related to attention processes. There is evidence that the Simon effect may occur relative to the position of the attentional focus (Nicoletti \& Umiltà, 1989) - more precisely, the shift of spatial attention that is made in temporal proximity with response selection (Nicoletti \& Umiltà, 1994; Rubichi, Iani, Nicoletti, \& Umiltà, 1997; Van der Lubbe \& Woestenburg, 1999). In addition, the Simon effect does not arise in the absence of spatial attention shifts (Nicoletti \& Umiltà, 1994; Notebaert, Soetens, \& Melis, 2001; Stoffer, 1991). Handedness has also been related to attention processes. From a historical perspective, the finding that the distance between a central fixation point and a movable point was overestimated in the right half of the visual field (Stevens \& Ducasse, 1912) led Smith (1914) to suggest that larger perceived objects in the right visual field attract visual attention, which, in turn, leads to grasping movements of the right hand. More recently, in his review, Peters (1995) emphasized that when both hands compete for attention, attention is asymmetrically distributed, favoring the right hand in right-handers (see, e.g., Peters, 1981). In addition, Peters (1995) also suggested that the attentional bias toward the right visual field would be the causal agent in hand preference. Rabbitt (1978) described an attentional bias toward the field of operation of the dominant hand. He required right- and left-handed participants to perform a reaching task in three different conditions: with the right hand only, with the left hand only, or choosing between hands. In the onehand conditions, no hand dominance effects were found. In contrast, when choices had to be made between hands, participants in both groups were faster with the dominant than with the nondominant hand. Interestingly, in the twohand condition the choice was based on stimulus position - that is, participants reached to the right when the stimulus was to the right and to the left when the stimulus was to the left. Rabbitt reasoned that handedness is a factor affecting the response selection stage and described it as an attentional bias toward one effector field - namely, that of the dominant hand.

To sum up, both the Simon effect and handedness are considered response selection phenomena with close links to attention-related processes. Thus, it seems reasonable to hypothesize that handedness may affect the way spatial coding takes place. From our own experience and from an initial informal assessment of the literature, we noted that when only right-handers are recruited the size of the Simon effect is often asymmetric - that is, it is greater in the right than in the left visual hemifield. We went through recent Simon studies to see whether the Simon effect is larger on the right side when participants are described as right-handed. For those studies in which the performance of normals was compared with that of pathological populations, we considered the data pertaining to the normal participants. In reviewing the literature, we considered only experiments published since 1990 in which a standard Simon task was used - that is, in which right and left bimanual responses were required for right and left visual stimuli. Of course, it is not possible to see asymmetries when mean RTs are collapsed in corresponding and noncorresponding trials. Asymmetries indeed become evident when mean RTs are divided by stimulus and response locations. Only five studies met the proposed criteria (see Table 1). In four of these studies, the data show that the Simon effect is larger in the right visual hemifield. Furthermore, there is evidence suggesting substantial differences in right-handers in the neural mechanisms that underlie Simon-effect-related response priming in the two cerebral hemispheres. In their transcranial magnetic stimulation study, Stürmer et al. (2000) used only right-handers and found that response priming processes evoked by stimulus location led to differential effects between hemispheres. Also, Angrilli, Zorzi, Tagliabue, Stegagno, and Umiltà (2001) studied right-handers and found right and left response asymmetries in both behavioral and psychophysiological data. 
Table 1

Studies With at Least One Experiment in Which a Standard Simon Task With Visual Stimuli and Bimanual Responses Was Used and Right-Handed Participants Were Selected

Study Presence of a Larger RSE

Gastaldo, Umiltà, Bianchin, \& Prior (2002)

Mandich, Buckolz, \& Polatajko (2002)

Nicoletti, Umiltà, \& Mapelli (1992)

Proctor \& Wang (1997)

Valle-Inclán (1996)

Note-The search of the literature was limited to studies published from 1990 to 2004. See text for details. In Mandich et al. (2002), the participants' handedness was not declared. However, data were analyzed by computing the interaction between hand preference (dominant vs nondominant) and Simon effect (corresponding vs. noncorresponding). Results showed that the Simon effect was markedly larger for the dominant than for the nondominant hand.

These data support the notion that handedness and spatial coding are closely related and that this relationship depends on an attentional bias toward the field of operation of the dominant hand. The main aim of the present work was to explore whether or not the Simon effect asymmetry described above is a reliable and systematic phenomenon and whether or not it is related to the participant's handedness (Experiment 1). From the studies reported above, we derived the prediction that the size of the Simon effect is dependent on the participant's handedness. More specifically, it should be larger in the right visual hemifield for right-handed participants and in the left visual hemifield for left-handed participants, whereas it should be symmetric for ambidextrous participants. A secondary aim was to show that the Simon effect asymmetry is due to an attentional bias toward the effector field in which the dominant hand is operating (Experiment 2).

Before we proceed, it is worth noting that there are three different patterns of data resulting from the interaction between stimulus and response locations that could produce the observed larger Simon effect for the right visual hemifield in right-handers. Figure 1 represents these three possibilities with hypothetical data. In panel A, the symmetric Simon effect is represented. Panel B represents the condition in which both an overall interaction between side and hand (indicative of the Simon effect) and a performance advantage for the dominant hand are present. In this case,
A

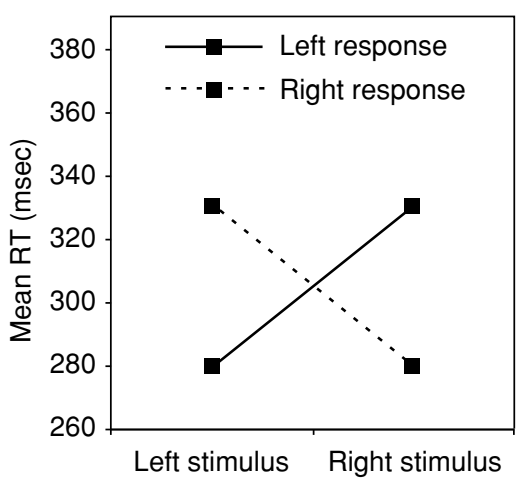

C

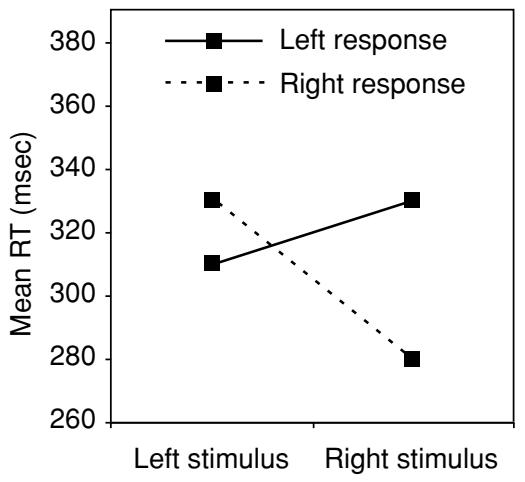

B

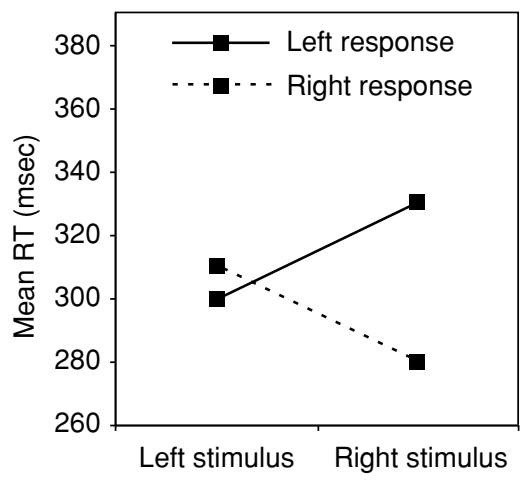

D

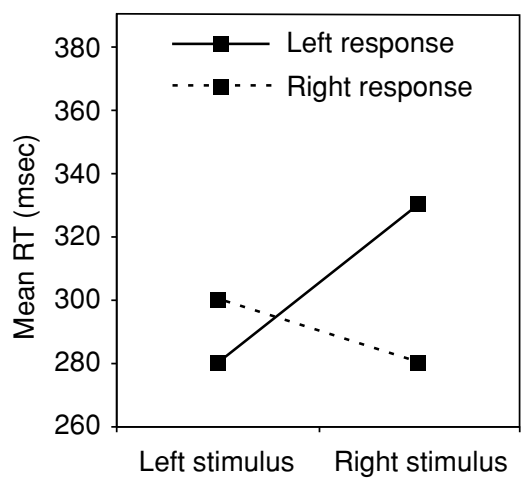

Figure 1. Data patterns of the interactions between stimulus and response locations are depicted with hypothetical data. Panel A shows the normal Simon effect, whereas in panels $B, C$, and $D$ the Simon effect is larger in the right visual hemifield. In panel $B$, the asymmetry depends on an unspecific advantage of the right hand. In panel $C$, the asymmetry depends on the advantage of the right hand in the corresponding space, whereas in panel $D$ it depends on the disadvantage of the left hand in the noncorresponding space. 
the Simon effect would be larger in the right visual hemifield and would depend on the performance advantage of the dominant hand. In other words, the asymmetric Simon effect could simply derive from an unspecific advantage of the dominant hand. This seems plausible given that there is a large body of evidence showing that handedness is associated with larger cortical representations (e.g., Triggs, Subramanium, \& Rossi, 1999) and a lower motor threshold (e.g., Triggs, Calvanio, \& Levine, 1997) for the dominant hand. Thus, different magnitudes of the Simon effect in the two visual hemifields per se may not be indicative of an interaction between handedness and spatial coding.

Panels C and D of Figure 1 represent the two conditions in which the asymmetric Simon effect depends on the interaction between handedness and spatial coding. There are two different data patterns that could produce this kind of asymmetry in right-handers. In the first, responses to right and left stimuli produce a larger RT difference for the right than for the left hand (panel C), whereas in the second it produces a larger RT difference for the left than for the right hand (panel D). These two data patterns suggest different mechanisms at the basis of an eventual asymmet- ric Simon effect. In the first case, the asymmetry would depend on an advantage of the dominant hand for stimuli presented in the corresponding space. The other situation would indicate a disadvantage of the nondominant hand when it operates in the noncorresponding space.

In Figure 2, we plotted the interaction between stimulus and response locations for the four experiments listed in Table 1 in which the Simon effect was larger in the right visual hemifield than in the left. As can be seen, the asymmetry arises from different data patterns. In the two experiments of Valle-Inclán (1996), as in Ivanoff (1998), the dominant hand responds faster in the corresponding space. On the contrary, the experiment of Proctor and Wang (1997) can be better interpreted as an example of the asymmetric Simon effect originated by an unspecific advantage of the dominant hand. The results of Nicoletti, Umiltà, and Mapelli (1992) could suggest that the asymmetric Simon effect could originate from the combination of the mechanisms explained above.

For the reasons we have presented above, in the present study we defined the Simon effect as asymmetric only in those cases in which it was (1) larger in one visual hemifield in the absence of an unspecific advantage of the
Nicoletti et al. (1992), Experiment 1

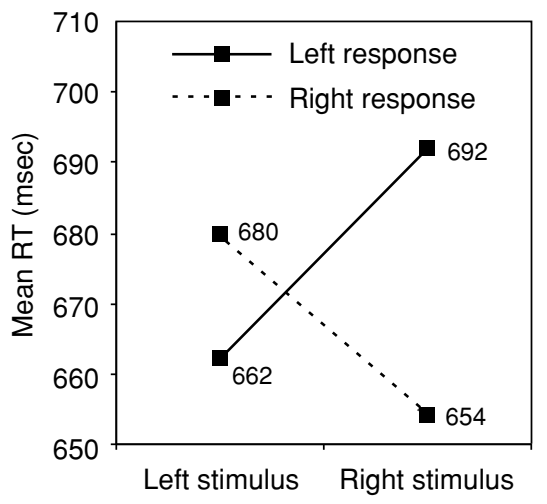

Valle-Inclán (1996), Experiment 2

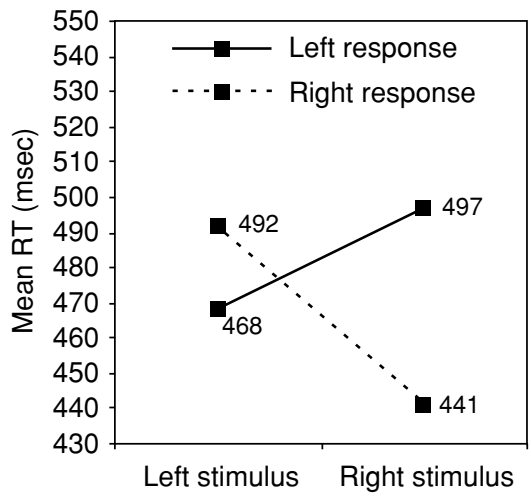

Valle-Inclán (1996), Experiment 1

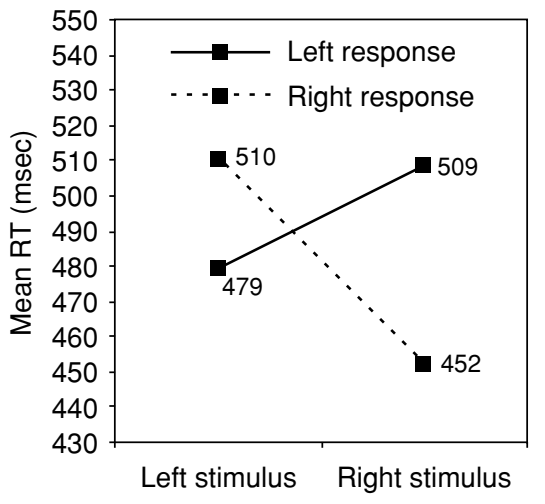

Proctor \& Wang (1997), Experiment 1 Neutral Condition

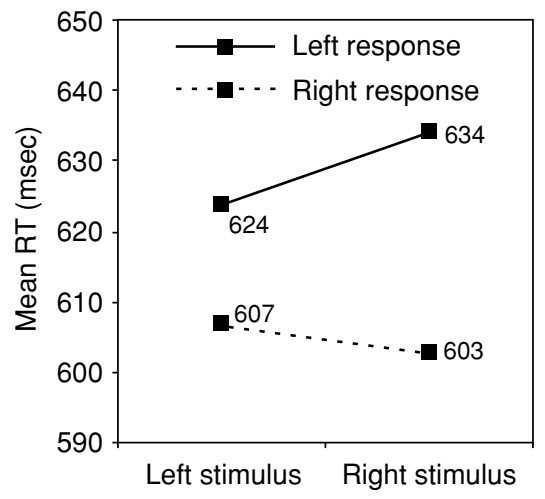

Figure 2. Data patterns of the interactions between stimulus and response locations of four experiments of the studies listed in Table 1. In the two experiments of Valle-Inclán (1996), the data were inferred from the plots. 
dominant hand and (2) present in addition to either faster responses for the corresponding dominant hand than for the corresponding nondominant hand, or slower responses for the noncorresponding nondominant hand than for the noncorresponding dominant hand.

\section{EXPERIMENT 1}

The purpose of the present experiment was to test whether or not the sizes of the left visual hemifield Simon effect (LSE) and of the right visual hemifield Simon effect (RSE) depended on handedness. For this purpose, righthanded, ambidextrous, and left-handed participants were asked to perform a standard color-discrimination Simon task. As stated above, we predicted that the RSE should be larger than the LSE in right-handed participants, whereas the opposite pattern should arise in left-handed participants. In addition, ambidextrous individuals should have a symmetric Simon effect- that is, the RSE and the LSE should be of the same magnitude.

\section{Method}

Participants. Hand preference was assessed according to the Edinburgh handedness inventory (Oldfield, 1971). We asked large classes of students of the University of Urbino to complete the inventory. ${ }^{2}$ Given that we were interested in comparing groups with different manual preferences, we divided the participants into three groups on the basis of handedness. We included in the study only participants with manual preferences of +80 to +100 (right-handed), -80 to -100 (left-handed), and -20 to +20 (ambidextrous). Then, we randomly selected for the experiment 20 right-handed, 20 ambidextrous, and 20 left-handed participants (10 males and 10 females in each group). All the participants had normal or corrected-to-normal visual acuity and were naive as to the purpose of the experiment.

Apparatus and Stimuli. The experiment took place in a dimly lit room. An IBM-compatible PC with an Intel 486 processor, controlled by Micro Experimental Laboratory (MEL, Version 2.0) software, was used. The participant sat in front of a 14-in. color CRT monitor screen on which the stimuli appeared. His or her head was positioned in an adjustable head- and chinrest so that the distance between the eyes and the center of the screen was of about $55 \mathrm{~cm}$. The stimuli (one green and one red $0.52^{\circ} \times 0.52^{\circ}$ square) could appear $4.3^{\circ}$ to the right or left of a fixation cross located at the center of the screen. The response keys were the rightmost and leftmost buttons of the MEL response box, which was located at the participant's body and computer midlines, and were pressed by the right and left index fingers, respectively.

Procedure. Each trial began with a central fixation cross, which remained on the screen throughout the trial. After $1 \mathrm{sec}$, a warning tone was presented for $100 \mathrm{msec}$. At its offset, a stimulus (either a red square or a green square) was randomly shown for $200 \mathrm{msec}$ to the left or right of fixation. The offset of the stimulus was followed by an $800-\mathrm{msec}$ response interval. At the end of each trial, feedback about RT (in the case of a correct response) or an error message (in the case of an incorrect response or no response) was displayed under the fixation cross for $500 \mathrm{msec}$. The intertrial interval was $1 \mathrm{sec}$.

The participants performed in one experimental session with five blocks of 60 trials each, the first block being for practice. There was a 2-3-min rest interval between blocks. Half of the participants of each group used the right button for the red square and the left button for the green square, whereas the other half had the reverse assignment. The participants were told to respond as quickly and accurately as possible.

\section{Results}

Only RTs from 100 to $900 \mathrm{msec}$ were included in the analyses. The overall error rate was $2.3 \%$. For each participant in each group, mean correct RTs were calculated as a function of corresponding and noncorresponding trials in the right and in the left visual hemifields. The results are summarized in Table 2.

Both errors and correct RTs were analyzed with ANOVAs with group (right-handed vs. ambidextrous vs. left-handed) as the between-subjects factor and visual hemifield (LSE vs. RSE) and Simon effect (corresponding vs. noncorresponding) as the within-subjects factors. Error analysis showed only a main effect of group $[F(2,57)=13.0$, $p<.001]$. Planned comparisons showed that the error rate of the right-handers $(0.9 \%)$ was significantly lower that those of both the ambidextrous $(2.8 \%)$ and the lefthanded participants (3.1\%). The RT analysis showed that there was on the whole a 20 -msec significant Simon effect $[F(1,57)=47.7, p<.001]$. More importantly, the threeway interaction was significant $[F(2,57)=4.0, p=.024]$. Planned comparisons showed that for the right-handed participants the RSE was significant ( $25 \mathrm{msec}, p=.001$ ) whereas the LSE was not (6 msec, $p=.266)$, the RSE being significantly greater than the LSE $(p=.025)$. In contrast, for the left-handed participants the RSE was not significant (14 msec, $p=.10)$ whereas the LSE was significant ( $31 \mathrm{msec}, p<.001)$. Also in the latter group, the difference between RSE and LSE was significant ( $p=$ $.05)$. The ambidextrous participants showed a symmetric Simon effect, with a significant RSE (22 msec, $p=.001)$ and a significant LSE (20 msec, $p=.022)$, which did not differ $(p=.785)$.

Also, as planned, $t$ tests were performed separately for the right-handed and left-handed groups to examine

Table 2

Mean Reaction Times (RTs, in Milliseconds) and Error Rates (ERs, in Percentages) for Corresponding (C) and Noncorresponding (NC) Trials, and Simon Effect (SE) in the Left Visual Hemifield (LVH) and the Right Visual Hemifield (RVH) for Each Group in Experiment 1

\begin{tabular}{|c|c|c|c|c|c|c|c|c|c|c|}
\hline \multirow[b]{3}{*}{ Group } & \multicolumn{5}{|c|}{ LVH } & \multicolumn{5}{|c|}{ RVH } \\
\hline & \multicolumn{2}{|c|}{$\mathrm{C}$} & \multicolumn{2}{|c|}{$\mathrm{NC}$} & \multirow[b]{2}{*}{ SE } & \multicolumn{2}{|c|}{$\mathrm{C}$} & \multicolumn{2}{|c|}{$\mathrm{NC}$} & \\
\hline & RT & ER & RT & ER & & $\mathrm{RT}$ & ER & RT & ER & $\mathrm{SE}$ \\
\hline Right-handed & 408 & 1.0 & 414 & 0.8 & 6 & 391 & 0.9 & 416 & 0.9 & 25 \\
\hline Ambidextrous & 382 & 2.6 & 402 & 2.6 & 20 & 384 & 2.8 & 406 & 3.2 & 22 \\
\hline Left-handed & 377 & 3.3 & 408 & 2.5 & 31 & 389 & 3.3 & 403 & 3.2 & 14 \\
\hline
\end{tabular}


whether the observed asymmetries depended on the difference in speed between right and left corresponding responses or on that between right and left noncorresponding responses (see Table 2 for the mean values). In the right-handers, corresponding right responses were significantly faster than corresponding left responses ( $p=$ $.003)$, whereas noncorresponding right and left responses did not differ significantly $(p=.68)$. In the left-handers, the difference between corresponding right and left responses was marginally significant $(p=.069)$, whereas noncorresponding right and left responses did not differ significantly $(p=.26)$.

To further examine the relations between handedness and the Simon effect in each visual hemifield, Pearson correlation coefficients were calculated between the Edinburgh inventory score and both the RSE and the LSE for all participants. The Edinburgh inventory showed a significant positive correlation with $\operatorname{RSE}(r=.53, p<.001)$ and a negative correlation with $\operatorname{LSE}(r=-.44, p=.004)$. Thus, the RSE increases and the LSE decreases with the increase of the Edinburgh inventory score, which is proportional to the degree of the participant's dexterity.

\section{Discussion}

In the present experiment, we examined whether or not the magnitude of the Simon effect in each visual hemifield is related to handedness. Results showed that the Simon effect was asymmetric for both the right-handed and the left-handed participants, whereas the ambidextrous individuals had a symmetric Simon effect. The right-handed participants had a significantly greater RSE than LSE, whereas the opposite was found for the lefthanded participants. The asymmetries were also qualified by the expected pattern of differences for corresponding and noncorresponding responses between dominant and nondominant hands. In particular, noncorresponding responses did not differ, whereas the difference between corresponding right and left responses was significant in right-handers and approached significance in lefthanders. The latter result can be explained by considering that left-handers tend to be a rather heterogeneous group in comparison with right-handers. In our sample, 12 of the 20 right-handers scored 90 or more on the Edinburgh inventory (10 of them reaching the maximum score), whereas only 3 left-handers scored less than -90 ( 1 of them reaching the minimum score). Given these considerations and the significant values of the Pearson correlation coefficients, it can be concluded that the present experiment showed a significant relationship between handedness and the Simon effect. The data patterns showed that in both groups the asymmetric Simon effect depended on the advantage of the dominant hand when it operates in the corresponding space. This indicates that at some stage of processing in corresponding trials, dominant handrelated processes are more efficient than nondominant hand-related processes.

Before we proceed, an alternative explanation needs to be considered. The magnitude of the Simon effect has been associated to the relative processing speed in the generation of irrelevant and relevant spatial codes (Hommel, 1993, 1994). According to this account, called the temporal overlap hypothesis, the irrelevant stimulus location code is formed rapidly upon stimulus presentation and then decays. Experimental manipulations that are supposed to reduce the degree of temporal overlap in the generation of the relevant and irrelevant codes would reduce the magnitude of the Simon effect. Indeed, manipulations that slow the identification of the relevant stimulus attribute, such as stimulus eccentricity, signal quality, signalbackground contrast, and certain features of the stimulus set, increase mean RTs and reduce the magnitude of the Simon effect.

A smaller Simon effect in the contralateral hemifield with respect to the dominant hand might therefore possibly be explained in terms of the temporal overlap. According to this view, right-handers would be slower than lefthanders in identifying the relevant information in the left visual field, whereas the opposite would be true for lefthanders. To test this possibility, we performed a post hoc ANOVA with group (right-handers vs. left-handers) as a between-subjects factor and stimulus position (right vs. left) as a within-subjects factor. The interaction was significant $[F(1,38)=9.13, p=.004]$. Planned comparisons showed that stimuli on the right were responded to faster (404 msec) than stimuli on the left (411 msec) only for the right-handed participants. The left-handers showed similar mean RTs for stimuli presented on the right and those presented on the left (396 vs. $393 \mathrm{msec}$, respectively). The present data do not support the temporal overlap explanation of the asymmetries in the Simon effect because the processing advantage in one visual hemifield was present in the right-handers only and its magnitude $(7 \mathrm{msec})$, though significant, was not large enough to explain the substantial difference in magnitude between RSE and LSE. Thus, it is unlikely that the asymmetry of the Simon effect was related to differences in the speed of processing for stimuli presented to the right and to the left in righthanders and left-handers.

\section{EXPERIMENT 2}

In the previous experiment, we found that the magnitudes of RSE and LSE varied as a function of handedness. The right-handed participants had a larger RSE, whereas the left-handed participants had a greater LSE. The asymmetries depended on an advantage of the dominant hand when it operated in the corresponding space. As we stated in the introduction, Rabbitt (1978) found that both righthanders and left-handers were faster with the dominant than with the nondominant hand in a reaching task. The hand to be used was determined on the basis of compatibility with the position (right or left) of an imperative stimulus (i.e., right-hand reaching with a right stimulus and left-hand reaching with a left stimulus). In Experiment 1 , we extended these results by using a choice RT task in which stimulus location was task irrelevant. Thus, the dominant-hand advantage seems to be a general phenomenon that arises in spatial coding tasks. 
We agree with Rabbitt (1978) that these findings might be explained by invoking an attentional bias in favor of the dominant-hand field of operation. There are two different ways to describe the field of operation of an effector. The first refers to the part of the space where the effector operates at a given moment, whereas the second refers to the part of the space where the effector usually operates. When the relevant effector is the hand, for example, it has been found that attentional effects are handcentered independently of where the hand is operating (Tipper, Lortie, \& Baylis, 1992). To corroborate the hypothesis that the dominant hand effect in spatial coding depends on a hand-centered attentional bias, in the present experiment we asked right-handed, left-handed, and ambidextrous participants to perform the same Simon-like color-discrimination task described for Experiment 1 with crossed hands (i.e., left hand on the right button and right hand on the left button). If the asymmetry of the Simon effect observed in Experiment 1 was due to a hand-centered attentional bias, then a reversed asymmetry should be obtained in right- and left-handed participants. In particular, the LSE should be larger than the RSE in right-handers and vice versa in left-handers. Alternatively, if the asymmetry depends on where the dominant hand usually operates, the same pattern of results as that found in Experiment 1 would be expected.

In addition, in the present experiment we were able to test in a finer grained manner whether or not the advantage of the dominant hand in its corresponding space depends on a more pronounced efficiency of the motor system in executing an already selected response with the dominant hand. As reported above, there is evidence that handedness is associated with a lower motor threshold of the dominant hand (see, e.g., Triggs et al., 1997). The results of Experiment 1 ruled out the possibility that this execution advantage exerts its effects independently of S-R trials. Indeed, the advantage of the dominant hand was present only on corresponding trials. It is possible, however, that the execution advantage of the dominant hand is neutralized during the conflict resolution process that takes place in noncorresponding trials. In other words, the asymmetries in the Simon effect may still depend on an advantage in the execution of responses with the dominant hand when these responses are automatically activated by stimulus location. ${ }^{3}$ If this is the case, the advantage of the dominant over the nondominant hand in corresponding trials should be more pronounced for fast responses - when the irrelevant stimulus location code is formed - and should decrease, or even disappear, for slow responses.

For this reason, in the present experiment response temporal dynamics were analyzed by means of bin distributional analyses (see, e.g., De Jong et al., 1994; Rubichi et al., 1997). ${ }^{4}$ Given that the Simon effect with crossed hands does not decrease along with increasing RTs, as the Simon effect with the hands in normal position does (Wascher, Schatz, Kuder, \& Verleger, 2001), the present experiment is more suitable than Experiment 1 for testing the kind of processing advantage of dominant hand for corresponding pairings. If there is an execution ad- vantage, we would expect to find it more pronounced for fast responding. On the contrary, if the advantage of the dominant hand in corresponding trials depends on timeconsuming attentional factors, we would expect it to be more pronounced for slow responses.

\section{Method}

Participants. Forty-eight new students of the University of Urbino were selected as in Experiment 1. Sixteen were right-handed, 16 ambidextrous, and 16 left-handed ( 8 males and 8 females in each group).

Apparatus, Stimuli, and Procedure. These were the same as those described for Experiment 1, the only exception being in regard to the response device. The response box buttons used for Experiment 1 would be uncomfortable with crossed hands because the two hands would largely overlap. Thus, in the present experiment the keyboard was used as the input device. In this manner, it was possible to use two keys that were farther away from each other. One key (" $z$ ") was located to the left of the body midline and was pressed by the right index finger, whereas the other key (")") was located to the right and was pressed by the left index finger.

\section{Results}

The error rate was $3.1 \%$. Only correct RTs in the same range as in Experiment 1 were considered for subsequent analyses. For each participant in each group, mean correct RTs were calculated as a function of corresponding and noncorresponding trials in the right and in the left visual hemifields. The RTs are summarized in Table 3.

The same analyses performed for Experiment 1 were performed for the present experiment. The error ANOVA did not yield any significant effect. The RT ANOVA showed that there was a significant Simon effect of $36 \mathrm{msec}[F(1,45)=125.3, p<.001]$. The three-way (group $\times$ visual hemifield $\times$ Simon effect) interaction was also significant $[F(2,45)=15.1, p<.001]$. Planned comparisons for the right-handers showed a nonsignificant RSE (13 msec, $p=.175$ ), a significant LSE (54 msec, $p<.001)$, and a significant difference between RSE and LSE $(p<.001)$. In the left-handers, both the RSE (49 msec, $p<.001)$ and the LSE $(20 \mathrm{msec}, p=.003)$ were significant and differed significantly $(p=.02)$. In the ambidextrous individuals, the two effects were very similar: a 39-msec RSE $(p<.001)$ and a 40-msec LSE $(p<.001)$.

For the right-handers, $t$ tests showed that corresponding left responses were faster than corresponding right responses $(p<.001)$ and noncorresponding left responses were significantly slower than noncorresponding right re-

Table 3

Mean Reaction Times (in Milliseconds) for Corresponding (C) and Noncorresponding (NC) Trials, and Simon Effect (SE) in the Left Visual Hemifield (LVH) and the Right Visual Hemifield (RVH) for Each Group in Experiment 2

\begin{tabular}{lccccccc}
\hline \multirow{2}{*}{ Group } & \multicolumn{3}{c}{ LVH } & & \multicolumn{3}{c}{ RVH } \\
\cline { 2 - 4 } \cline { 6 - 8 } & C & NC & SE & & C & NC & SE \\
\hline Right-handed & 386 & 440 & 54 & & 413 & 426 & 13 \\
Ambidextrous & 400 & 440 & 40 & & 399 & 438 & 39 \\
Left-handed & 395 & 415 & 20 & & 382 & 431 & 49 \\
\hline
\end{tabular}


sponses $(p<.001)$. In the left-handers, $t$ tests showed that corresponding right responses were significantly faster than corresponding left responses ( $p=.049)$ and noncorresponding right responses were slower than noncorresponding left responses ( $p=.044)$.

Two separate bin distributional analyses were performed for the right-handed and left-handed groups. The first, with Simon effect (corresponding vs. noncorresponding) and bin (first through fifth) as within-subjects factors, was aimed at showing the temporal dynamics of the LSE and RSE for right- and left-handers, respectively. The second bin distributional analysis was performed to determine the difference between the dominant and nondominant hands in corresponding trials. In this analysis, hand (dominant vs. nondominant) and bin were the within-subjects factors. Since what matters for the purpose of the present experiment is the interaction between factors, only the data relative to the interaction is reported.

With regard to the right-handers, the first bin distributional analysis showed a significant interaction between the Simon effect and bin $[F(4,60)=5.69, p=.001]$. Pairwise comparisons showed that the Simon effect was always significant $(p \mathrm{~s}<.006)$ from the first through the fifth bins $(56,62,62,57$, and $31 \mathrm{msec}$, respectively) and that the Simon effect for the slowest bin was smaller than that for the other bins $(p s<.02)$ except for the first bin. In the bin distributional analysis for corresponding pairings, the hand $\times$ bin interaction was significant $[F(4,60)=$ $4.53, p=.003]$. Pairwise comparisons showed that the difference between nondominant and dominant hand responses was small and nonsignificant in the first bin (11 msec, $p=.10)$, whereas it was greater and significant in the second through fifth bins $(23,29,32$, and $39 \mathrm{msec}$, respectively, $p \mathrm{~s}<.002$ ).

With regard to the left-handers, the interaction between the Simon effect and bin was not significant (the Simon effect values from the first through the fifth bins were 38, 50, 56,56 , and $42 \mathrm{msec}$, respectively). In the bin distributional analysis for corresponding pairing, the hand $\times$ bin interaction was not significant. The differences between nondominant and dominant hand responses were 9, 9, 12, 15, and $17 \mathrm{msec}$ from the first to the fifth bins, respectively.

\section{Discussion}

In the present experiment, we examined whether the asymmetry of the Simon effect in right- and left-handers was related to the position where the dominant hand operates, as predicted by the hand-centered attentional bias hypothesis. The only difference with respect to Experiment 1 was that here the participants responded with crossed hands. Results showed exactly the opposite pattern found in Experiment 1-that is, RSE was larger than LSE in the left-handed participants whereas LSE was larger than RSE in the right-handed participants. As in Experiment 1, the asymmetries were qualified by the expected pattern of differences for corresponding and noncorresponding responses between dominant and nondominant hands, and the ambidextrous participants showed a symmetric Simon effect.
The analysis of the time course of response conditions as indexed by the bin distributional analyses showed that the Simon effect in the visual hemifield in which the dominant hand is operating is rather constant over time. In addition, the advantage of the dominant over the nondominant hand for corresponding trials increased along with response speed. The latter results seem to rule out an explanation in terms of an execution advantage for the dominant hand in corresponding pairings and support the notion of an attentional advantage of the dominant hand. On the whole, these results seem to support the view that the asymmetry in the magnitude of the Simon effect depends on an attentional bias that is hand-centered, and to rule out the possibility that there is an attentional bias toward the hemispace where the dominant hand normally operates.

An unexpected outcome of the present study was that the overall magnitude of the Simon effect was rather different between Experiments 1 and 2 (19 vs. $36 \mathrm{msec}$, respectively). We performed a post hoc one-way ANOVA on the size of the Simon effect with experiment as a betweensubjects factor. Unlike in previous studies in which the Simon effect was of the same magnitude with crossed and with uncrossed hands (e.g., Roswarski \& Proctor, 1996; Wallace, 1971), a significant main effect of experiment was found in our study $[F(1,106)=14.2, p=.0003]$. One possible reason for this difference in the magnitude of the effect might rely on the response devices used in Experiments 1 and 2 (the MEL response box and the keyboard, respectively). Whereas on the MEL response box the distance between the two response buttons was $8 \mathrm{~cm}$, it was $17 \mathrm{~cm}$ on the keyboard. There is evidence that the distance between the two responses is an important variable for determining the salience of the distinction between right and left locations (see the relative salience account-e.g., Proctor, $\mathrm{Vu}, \&$ Nicoletti, 2003). In other words, it is likely that in Experiment 2 the Simon effect was greater than in Experiment 1 because the distance between the two response buttons was greater, thus rendering more salient the distinction between right and left responses.

\section{GENERAL DISCUSSION}

The aim of the present study was twofold. First, we wanted to examine whether or not the magnitude of the Simon effect in the right and left visual hemifields is dependent on handedness. Experiment 1 showed clearly that the Simon effect was symmetric in the ambidextrous participants, whereas the RSE was larger in the right-handers and the LSE was larger in the left-handers. Second, we wanted to test whether or not this phenomenon can be explained by invoking an attentional bias centered on the position of the dominant hand. For this purpose, in Experiment 2 right-handers, left-handers, and ambidextrous participants performed the same Simon task as in Experiment 1 but with crossed hands. This manipulation reversed the pattern of results obtained in Experiment 1-that is, the RSE was larger in the left-handers and the LSE was larger in the right-handers. In both experiments, dominant hand 
responses were faster than nondominant hand responses only in the case of corresponding pairings, whereas they were as fast as (or even slower than) nondominant hand responses for noncorresponding pairings. Thus, it seems unlikely that the asymmetries are due to an unspecific advantage of dominant hand responses. Experiment 2 ruled out the possibility that the advantage for the dominant hand in corresponding pairings is caused by an execution advantage, given that it was more pronounced for slower than for faster responses. Likewise, these results support the idea that the advantage depends on spatial attentional processes that require time to take place and indicate that the direct route of the response selection machinery for the dominant hand is more efficient than that of the nondominant hand. In addition, results from Experiment 2 suggest that the asymmetric Simon effect observed in relation to handedness depends on the field of operation in which the dominant hand operates at a given moment. As we have already stated, Rabbitt (1978) ascribes the handedness effect to response selection operations and considers it as an attentional bias toward the field in which the dominant hand operates.

This conclusion suggests that there are close links between the attentional and the motor systems and is in accordance with a prominent theory that considers spatial attention as the fundamental mechanism for the selection of a specific action directed toward a target object (Allport, 1987). According to a related account known as the premotor theory of attention, the facilitation of processing due to attention is a consequence of activation of neural circuits involved in motor preparation (Rizzolatti, Riggio, Dascola, \& Umiltà, 1987). On both accounts, rather than considering perception and action as separate functional and brain systems, it is more useful to consider a perception-for-action system in which the role of attention is to select one goal-directed action from among all possible actions that can be selected at a given time. There is indeed evidence that certain neural systems for motor control, such as those for eye and hand movements, show cross-talk effects (see, e.g., Tipper, Howard, \& Paul, 2001) and are influenced by spatial attention (see, e.g., Sheliga, Craighero, Riggio, \& Rizzolatti, 1997). In addition, physiologic studies with monkeys (e.g., Andersen, 1995; Graziano \& Gross, 1995) and experimental work with humans (e.g., Soechting \& Flanders, 1989) indicate that visual information can be coded with respect to a number of reference frames (e.g., retinotopic, head centered, shoulder centered, hand centered) that originate at the locus of the effector (e.g., eye, head, shoulder, hand, respectively) used to "acquire" the target. These findings led to the notion of action-centered attention. As we stated above, when the relevant effector is the hand, attention is hand centered (Tipper et al., 1992).

Given that the dominant hand has a larger cortical representation than the nondominant hand in both right- and left-handers (see, e.g., Triggs et al., 1999), we suggest that the dominant hand attentional bias effect is the behavioral manifestation of the larger cortical representation of the perception-for-action system of the dominant hand. Spatial attention processes related to the dominant hand would be more efficient than those related to the nondominant hand. This claim is in accordance with the hypothesized causal relation of spatial attention for handedness put forward by Peters (1995). As we stated above, there is evidence that in Simon-like tasks spatial attention is one of the mechanisms used to split the space in right and left portions (see Stoffer \& Umiltà, 1997, for reviews). In other words, the spatial codes of spatially located S-R events depend on the shifts of spatial attention in the visual space. The present works suggest that spatial attention shifts are biased toward the part of the space where the dominant effector is operating. Given that the Simon effect depends on the strength of the spatial codes and that spatial codes are determined, at least in part, by the spatial attention shifts, the Simon effect is larger for the hemifield where the dominant hand is operating at a given moment.

Important insights into how attentional bias originating from the position of the dominant hand might operate can be drawn from the study of brain-injured patients with unilateral left neglect. These patients fail to report or act on stimuli on the left side of body-centered (egocentric) space, or to stimuli to the relative (allocentric) left of other stimuli. Usually, neglect arises in right-handers with right brain damage to the posterior attentional system that controls spatial attention (Posner \& Petersen, 1990). More precisely, according to the attention accounts described above, neglect phenomena have been attributed to an asymmetry in action selection processes. The basic idea is that selection and motor preparation for action are biased toward stimuli on the right (or relative right), and stimuli further to the left exert less influence on selection processes (Mattingley \& Driver, 1996). Interestingly, it has been found that the position of the right hand is a relevant factor in determining the left-right asymmetry in neglect patients. Usually, neglect patients fail to report light touches in the contralesional hand when their hands are in the anatomic situation that is in their corresponding space, whereas performance with the ipsilesional hand is generally similar to that of normal control participants. The contralesional deficit is reduced when patients have their hands crossed (Aglioti, Smania, \& Peru, 1999; Smania \& Aglioti, 1995), suggesting that the pathologic attentional bias toward the right side can be partially counterbalanced by the dominant hand position. It has been found that when neglect patients are required to reach with the right hand toward a target starting from right and left positions in the presence of distractors, distractors in the corresponding allocentric and egocentric locations are neglected (and thus cause very little or no interference) when they are located to the relative left of the hand. On the contrary, as with normal participants (Tipper et al., 1992), when distractors are to the relative right of the hand, they show interference (Buxbaum \& Permaul, 2001; Husain, Mattingley, Rorden, Kennard, \& Driver, 2000).

To sum up, there is evidence in both normal participants and brain-injured patients of an attentional bias centered on the dominant hand. We tentatively suggest here that 
the dominant-hand-centered attentional bias effect is the behavioral manifestation of a larger cortical representation of the dominant hand in both right- and left-handed participants.

\section{REFERENCES}

Aglioti, S., Smania, N., \& Peru, A. (1999). Frames of reference for mapping tactile stimuli in brain-damaged patients. Journal of Cognitive Neuroscience, 11, 67-79.

Allport, A. (1987). Selection for action: Some behavioral and neurophysiological considerations of attention and action. In H. Heuer \& A. F. Sanders (Eds.), Perspectives on perception and action (pp. 395419). Hillsdale, NJ: Erlbaum.

ANDERSEN, R. A. (1995). Coordinate transformations and motor planning in posterior parietal cortex. In M. S. Gazzaniga (Ed.), The cognitive neurosciences (pp. 519-532). Cambridge, MA: MIT Press.

Angrilli, A., Zorzi, M., Tagliabue, M., Stegagno, L., \& Umiltà, C. (2001). Cortical plasticity of spatial stimulus-response associations: Electrophysiological and behavioral evidence. NeuroReport, 12, 973977.

Buxbaum, L. J., \& Permaul, P. (2001). Hand-centered attentional and motor asymmetries in unilateral neglect. Neuropsychologia, 39, 653664.

De Jong, R., Liang, C.-C., \& Lauber, E. (1994). Conditional and unconditional automaticity: A dual-process model of effects of spatial stimulus-response correspondence. Journal of Experimental Psychology: Human Perception \& Performance, 20, 731-750.

EIMER, M. (1995). Stimulus-response compatibility and automatic response activation: Evidence from psychophysiological studies. Journal of Experimental Psychology: Human Perception \& Performance, 21, 837-854.

Gastaldo, S., Umiltà, C., Bianchin, G., \& Prior, M. (2002). The Simon effect in schizophrenic patients with negative symptoms. Cortex, 38, 149-159.

Graziano, M. S. A., \& Gross, C. G. (1995). The representation of extrapersonal space: A possible role for bimodal, visual-tactile neurons. In M. S. Gazzaniga (Ed.), The cognitive neurosciences (pp. 10211034). Cambridge, MA: MIT Press.

Hasbroucq, T., Possamaï, C.-A., Bonnet, M., \& Vidal, F. (1999). Effect of the irrelevant location of the response signal on choice reaction time: An electromyographic study in humans. Psychophysiology, 36, 522-526.

Hommel, B. (1993). The relationship between stimulus processing and response selection: Evidence for a temporal overlap. Psychological Research, 55, 280-290.

Hommel, B. (1994). Spontaneous decay of response code activation. Psychological Research, 56, 261-268.

Hommel, B., \& Prinz, W. (EDs.) (1997). Theoretical issues in stimulusresponse compatibility. Amsterdam: North-Holland.

Husain, M., Mattingley, J. B., Rorden, C., Kennard, C., \& Driver, J. (2000). Distinguishing sensory and motor biases in parietal and frontal neglect. Brain, 123, 1643-1659.

IVANOFF, J. (1998). Left- and right-handers distribute their attention asymmetrically on stimulus-response compatibility tasks. Unpublished master's thesis, University of Guelph, Guelph, Ontario, Canada.

Kornblum, S., Hasbroucq, T., \& Osman, A. (1990). Dimensional overlap: Cognitive basis for stimulus-response compatibility-A model and taxonomy. Psychological Review, 97, 253-270.

Lamberts, K., Tavernier, G., \& D'Ydewalle, G. (1992). Effects of multiple reference points in spatial stimulus-response compatibility. Acta Psychologica, 79, 115-130.

Lu, C.-H., \& Proctor, R. W. (1995). The influence of irrelevant location information on performance: A review of the Simon and spatial Stroop effects. Psychonomic Bulletin \& Review, 2, 174-207.

Mandich, A., Buckolz, E., \& Polatajko, H. (2002). On the ability of children with developmental coordination disorder (DCD) to inhibit response initiation: The Simon effect. Brain \& Cognition, 50, 150-162.

Mattingley, J. B., \& Driver, J. (1996). Distinguishing sensory and motor deficits after parietal damage: An evaluation of response selection biases in unilateral neglect. In P. Thier \& H.-O. Karnath (Eds.), Parietal lobe contributions to orientation in 3D space (pp. 309-338). Heidelberg: Springer.

Nicoletti, R., \& Umiltà, C. (1989). Splitting visual space with attention. Journal of Experimental Psychology: Human Perception \& Performance, 15, 164-169.

Nicoletti, R., \& Umiltà, C. (1994). Attention shifts produce spatial stimulus codes. Psychological Research, 56, 144-150.

Nicoletti, R., Umiltà, C., \& Mapelli, D. (1992). Spatial representations of words and nonwords. Cortex, 28, 163-174.

Notebaert, W., Soetens, E., \& Melis, A. (2001). Sequential analysis of a Simon task: Evidence for an attention-shift account. Psychological Research, 65, 170-184.

OLDFIELD, R. C. (1971). The assessment and analysis of handedness: The Edinburgh inventory. Neuropsychologia, 9, 97-113.

Peters, M. (1981). Attentional asymmetries during concurrent bimanual performance. Quarterly Journal of Experimental Psychology, 33, 95-103.

Peters, M. (1995). Handedness and its relation to other indices of cerebral lateralization. In R. J. Davidson \& K. Hugdahl (Eds.), Brain asymmetry (pp. 183-214). Cambridge, MA: MIT Press.

Posner, M. I., \& Petersen, S. E. (1990). The attention system of the human brain. Annual Review of Neuroscience, 13, 25-42.

Proctor, R. W., Vu, K.-P. L., \& Nicoletti, R. (2003). Does right-left prevalence occur for the Simon effect? Perception \& Psychophysics, 65, 1318-1329.

Proctor, R. W., \& WANG, H. (1997). Enhancement of the Simon effect by response-location precues: Evaluation of the stimulus-identification account. Acta Psychologica, 95, 279-298.

RABBITT, P. (1978). Hand dominance, attention, and the choice between responses. Quarterly Journal of Experimental Psychology, 30, 407416

Rizzolatti, G., Riggio, L., Dascola, I., \& Umiltà, C. (1987). Reorienting attention across the horizontal and vertical meridians: Evidence in favor of a premotor theory of attention. Neuropsychologia, 25, 31-40.

Roswarski, T. E., \& Proctor, R. W. (1996). Multiple spatial codes and temporal overlap in choice-reaction tasks. Psychological Research, 59, 196-211.

Rubichi, S., Iani, C., Nicoletti, R., \& Umiltà, C. (1997). The Simon effect occurs relative to the direction of an attention shift. Journal of Experimental Psychology: Human Perception \& Performance, 23, 1353-1364

Sheliga, B. M., Craighero, L., Riggio, L., \& Rizzolatti, G. (1997). Effect of spatial attention on directional manual and ocular responses. Experimental Brain Research, 114, 339-351.

Simon, J. R., \& Rudell, A. P. (1967). Auditory S-R compatibility: The effect of an irrelevant cue on information processing. Journal of Applied Psychology, 51, 300-304.

Smania, N., \& Aglioti, S. (1995). Sensory and spatial components of somaesthetic deficits following right brain damage. Neurology, 45, 1725-1730.

Smith, S. (1914). Right and left handedness. Psychological Bulletin, 11, 400-402.

Soechting, J. F., \& Flanders, M. (1989). Sensorimotor representations for pointing to targets in three-dimensional space. Journal of Neurophysiology, 62, 582-594.

Stevens, H. C., \& Ducasse, C. J. (1912). The retina and right-handedness Psychological Review, 19, 1-31.

Stoffer, T. H. (1991). Attentional focussing and spatial stimulusresponse compatibility. Psychological Research, 53, 127-135.

Stoffer, T. H., \& Umiltà, C. (1997). Spatial stimulus coding and the focus of attention in S-R compatibility and the Simon effect. In B. Hommel \& W. Prinz (Eds.), Theoretical issues in stimulus-response compatibility (pp. 181-208). Amsterdam: North-Holland

Stürmer, B., Siggelkow, S., Dengler, R., \& Leuthold, H. (2000). Response priming in the Simon paradigm: A transcranial magnetic stimulation study. Experimental Brain Research, 135, 353-359.

Tipper, S. P., Howard, L. A., \& Paul, M. A. (2001). Reaching affects saccade trajectories. Experimental Brain Research, 136, 241-249.

Tipper, S. P., Lortie, C., \& BAYlis, C. G. (1992). Selective reaching: 
Evidence for action-centered attention. Journal of Experimental Psychology: Human Perception \& Performance, 18, 891-905.

Triggs, W. J., Calvanio, R., \& Levine, M. (1997). Transcranial magnetic stimulation reveals a hemispheric asymmetry correlate of intermanual differences in motor performance. Neuropsychologia, $\mathbf{3 5}$, 1355-1363.

Triggs, W. J., Subramanium, B., \& Rossi, F. (1999). Hand preference and transcranial magnetic stimulation asymmetry of cortical motor representation. Brain Research, 835, 324-329.

Umiltà, C., \& LiotTi, M. (1987). Egocentric and relative spatial codes in S-R compatibility. Psychological Research, 49, 81-90.

Umiltà, C., \& Nicoletti, R. (1985). Attention and coding effects in S-R compatibility due to irrelevant spatial cues. In M. I. Posner \& O. S. M. Marin (Eds.), Attention and performance XI (pp. 457-471). Hillsdale, NJ: Erlbaum.

VALLE-INCLÁN, F. (1996). The locus of interference in the Simon effect: An ERP study. Biological Psychology, 43, 147-162.

Van der Lubbe, R. H. J., \& Woestenburg, J. C. (1999). The influence of peripheral precues on the tendency to react towards a lateral relevant stimulus with multiple-item arrays. Biological Psychology, 51, 1-21.

WALLACE, R. J. (1971). S-R compatibility and the idea of a response code. Journal of Experimental Psychology, 88, 354-360.

WALLACE, R. J. (1972). Spatial S-R compatibility effects involving kinesthetic cues. Journal of Experimental Psychology, 93, 163-168.
Wascher, E., Schatz, U., Kuder, T., \& Verleger, R. (2001). Validity and boundary conditions of automatic response activation in the Simon task. Journal of Experimental Psychology: Human Perception \& Performance, 27, 731-751.

\section{NOTES}

1. To our knowledge, there is only one study in which the performance on the Simon task of right-handers was directly compared with that of left-handers. Ivanoff (1998) found that for right-handers right responses to rightward stimuli tended to be faster than left responses to leftward stimuli, whereas the opposite was true for left-handers.

2 . The data of the present work were collected when the authors were at the University of Urbino.

3 . We thank Jason Ivanoff for suggesting this possibility.

4. In the bin distributional analysis, RTs for each participant are ranked from shortest to longest in each condition as a function of correspondence and are usually divided into five bins, for each of which a mean is obtained. This procedure allows one to explore differences between response conditions as a function of response speed. For example, it is possible to obtain Simon effects for each bin by subtracting corresponding RT from noncorresponding RT.

(Manuscript received June 27, 2003;

revision accepted for publication September 30, 2005.) 